
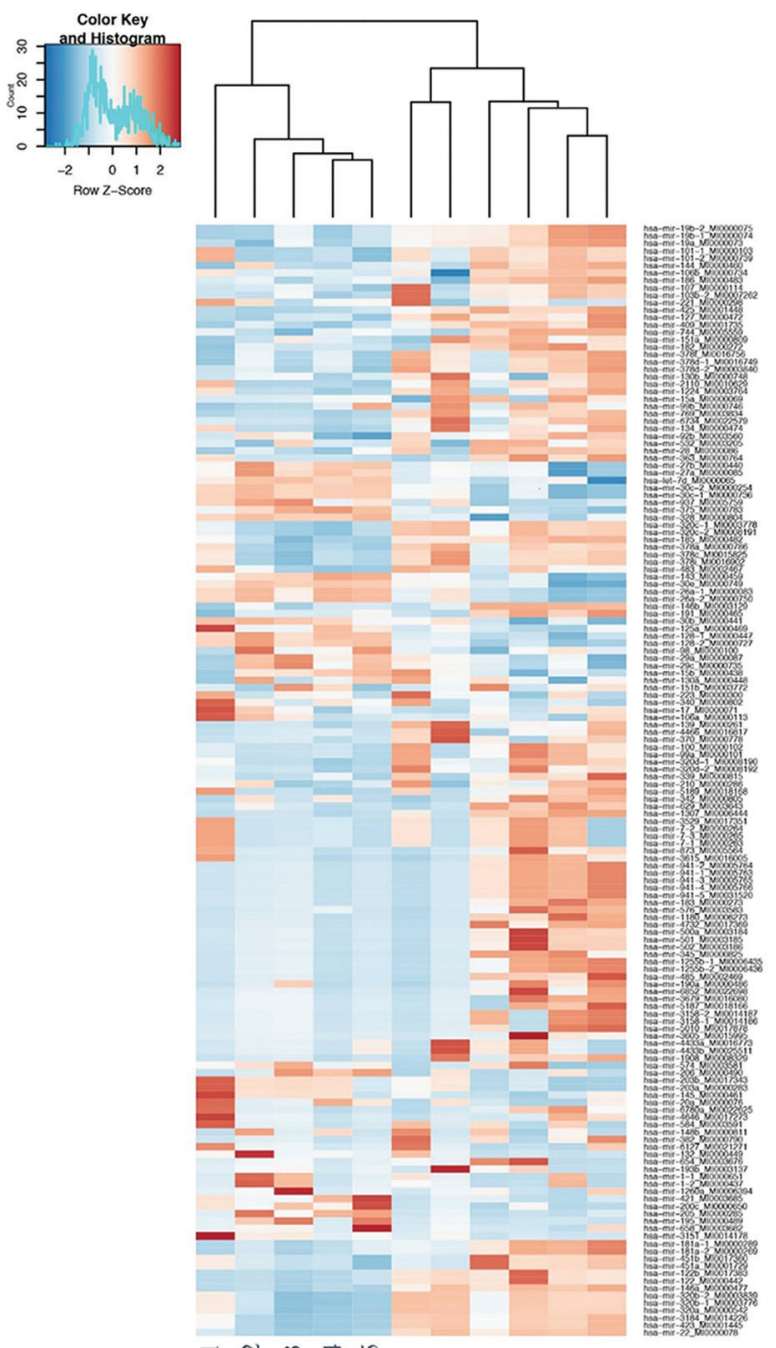

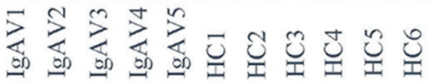

Figure 1. Heatmap based clustering analysis of serum miRNA expression

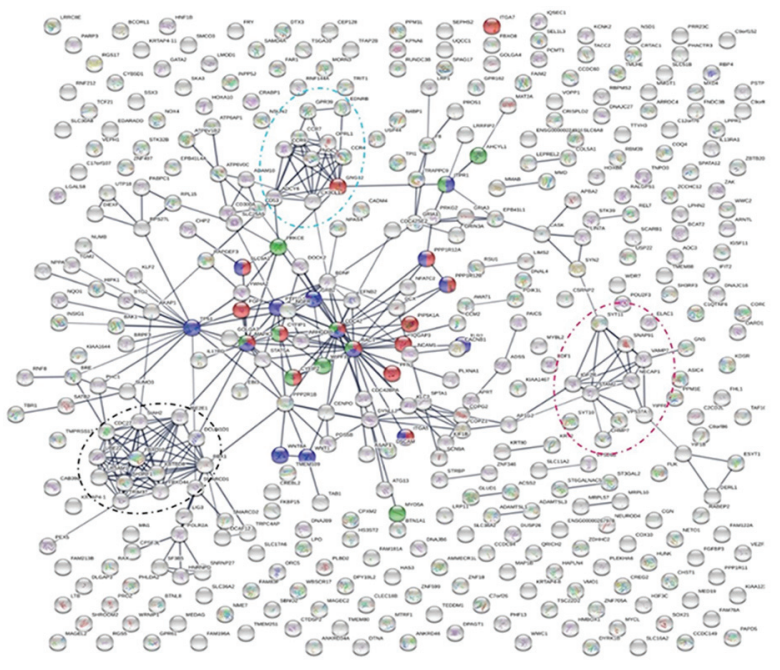

Color key: Regulation of actin cytoskeleton, Proteoglycans in cancer, Fcgamma receptor mediated phagocytosis, Chemokines and their receptors, Ubiquitination process, Vesicle, endocytosis, lysosomes and their trafficking

Figure 2. STRING Protein networks of gene targets of DE miRNAs $\left.\mathrm{b} / \mathrm{c}, \quad-378 \mathrm{a},-423,-3184 ; \mathrm{p}_{\mathrm{adj}}<10^{-10}\right)$, whereas 60 miRNAs were decreased (e.g. miRNA-128, -937, -30c, -328, -26a, $p_{\text {adj }}<0.005$ ), thereby representing the IgAV-associated serum miRNA signature. The miRror platform identified 426 protein-coding genes as targets of the $54 \mathrm{DE}$ miRNAs (log2 fold change $\geq|1|, p_{a d j}<0.01$ ). As analyzed by STRING, these miRNA target genes were enriched in distinct molecular networks and biological pathways, including "Phosphoproteins (FDR $7.4^{*} 10^{-5}$ ), "Alternative splicing" (FDR $2.8^{*} 10^{-4}$ ), "Regulation of actin cytoskeleton" (FDR 0.008 ), "Proteoglycans in cancer" (FDR 0.009) and "Fcgamma receptor dependent phagocytosis" (FDR 0.01) (Figure 2). Additionally, miRNA gene targets formed three distinct molecular clusters, specifically "Chemokines and their receptors", "Ubiquitination process" and "Vesicle, endocytosis, lysosomes and their trafficking" (Figure 2). This suggests that the altered miRNAs in IgAV regulate diverse cellular functions, reflecting or contributing to the key aspects of disease pathogenesis.

Conclusion: Here we report for the first time an IgAV-associated serum miRNA signature. The altered miRNAs clearly discriminate $\lg A \mathrm{~V}$ patients from $\mathrm{HC}$, and might play a key role in the pathogenesis of IgAV. Our study sets the basis for the identification of novel, serum-based disease biomarkers in vasculitis.

Disclosure of Interests: ALOJZIJA HOCEVAR: None declared, Katja Lakota: None declared, Thomas Grentzinger: None declared, Alexis Sarazin: None declared, Neža Brezovec: None declared, Tadeja Kuret: None declared, Oliver Distler Grant/research support from: Prof. Distler received research funding from Actelion, Bayer, Boehringer Ingelheim and Mitsubishi Tanabe to investigate potential treatments of scleroderma and its complications, Consultant for: Prof. Distler has/had consultancy relationship within the last 3 years with Actelion, AnaMar, Bayer, Boehringer Ingelheim, ChemomAb, espeRare foundation, Genentech/Roche, GSK, Inventiva, Italfarmaco, iQvia, Lilly, medac, Medlmmune, Mitsubishi Tanabe Pharma, Pharmacyclics, Novartis, Pfizer, Sanofi, Serodapharm and UCB in the area of potential treatments of scleroderma and its complications. In addition, he had/has consultancy relationship within the last 3 years with A. Menarini, Amgen, Abbvie, GSK, Mepha, MSD, Pfizer and UCB in the field of arthritides and related disorders, Sasa Cucnik: None declared, Olivier Voinnet: None declared, Snežna Sodin-Šemrl: None declared, Matija Tomšič: None declared, Mojca Frank-Bertoncelj : None declared DOI: 10.1136/annrheumdis-2019-eular.6874

\section{SAT0235 BIOLOGICAL THERAPYIN NEUROBEHÇET. MULTICENTER STUDY OF 29 PATIENTS}

José Luis Martín-Varillas ${ }^{1}$, Iñigo González-Mazón ${ }^{1}$, Belén Atienza-Mateo ${ }^{1}$, Monica Calderón-Goercke ${ }^{1}$, D. Prieto-Peña ${ }^{1}$, Lara Sánchez Bilbao ${ }^{1}$, Vanesa Calvo-Río ${ }^{1}$, Santos Castañeda ${ }^{2}$, Esther Vicente ${ }^{2}$, Olga Maiz ${ }^{3}$, Clara Moriano ${ }^{4}$, Elvira Diez Alvarez ${ }^{4}$, José Luis Andréu Sánchez ${ }^{5}$, Concepción Delgado Beltrán ${ }^{6}$, Marta Loredo Martínez ${ }^{6}$, J. Narváez ${ }^{7}$, Angel Ramos Calvo ${ }^{8}$, Francisca Sivera ${ }^{9}$, Enrique Raya ${ }^{10}$, Norberto Ortego $^{10}$, Jose Luis Callejas-Rubio ${ }^{10}$, Anahy Brandy-Garcia ${ }^{11}$, Alejandro Olive ${ }^{11}$, Sabela Fernández ${ }^{12}$, Ricardo Gómez de la Torre ${ }^{13}$, Ignacio Torre-Salaberri ${ }^{14}$, Julio Sánchez ${ }^{15}$, Ana UrruticoecheaArana $^{16}$, Eva Salgado-Pérez ${ }^{17}$, Rafael Melero ${ }^{18}$, Olga Martínez González ${ }^{19}$, Susana Romero-Yuste ${ }^{20}$, Miguel A. González-Gay', Ricardo Blanco ${ }^{1}{ }^{1}$ H.U.M. Valdecilla, Santander, Spain; ${ }^{2} \mathrm{H}$. La Princesa, Madrid, Spain; ${ }^{3} \mathrm{H}$. Donosti, San Sebastián, Spain; ${ }^{4} H$. de León, León, Spain; ${ }^{5}$ H. Puerta de Hierro, Madrid, Spain; ${ }^{6}$ H. Lozano Blesa, Zaragoza, Spain; ${ }^{7}$ H. Bellvitge, Barcelona, Spain; ${ }^{8}$ H. de Soria, Soria, Spain; ${ }^{9} \mathrm{H}$. de Elda, Alicante, Spain; ${ }^{10} \mathrm{H}$. San Cecilio, Granada, Spain; ${ }^{11} \mathrm{H}$. Germans Trias i Pujol, Barcelona, Spain; ${ }^{12}$ H. San Agustín, Avilés, Spain; ${ }^{13}$ H.U.C. Asturias, Oviedo, Spain; ${ }^{14} \mathrm{H}$. Basurto, Bilbao, Spain; ${ }^{15} \mathrm{H} .12$ de Octubre, Madrid, Spain; ${ }^{16} \mathrm{H}$. Can Misses, Ibiza, Spain; ${ }^{17} \mathrm{H}$. Ourense, Ourense, Spain; ${ }^{18} \mathrm{H}$. Vigo, Vigo, Spain; ${ }^{19} \mathrm{H}$. Salamanca, Salamanca, Spain; ${ }^{20} \mathrm{H}$. Pontevedra, Pontevedra, Spain

Background: Behçet's disease $(B D)$ is a variable vessel vasculitis and typically presents with mucocutaneous involvement. However, any organ can be affected, being the neurological affectation (neurobehçet, NB) one of the most serious manifestations.

Objectives: Our aim was to assess the efficacy and safety of biological therapy as treatment of NB.

Methods: We set up a multicenter observational study of 29 patients with NB on treatment with biological therapy (BT). NB diagnosis was made by neuroimaging, CSF analysis and/or suggestive clinical signs of central and/or peripheral nervous system involvement, excluding infectious causes or more prevalent pathology. Results are expressed as mean $\pm S D$ or as median and interquartile range (IQR) as appropriate.

Results: 29 patients $\left(15 \sigma^{x} / 14 \%\right)$ with an average age of $39.6 \pm 10.5$ years. HLA-B51 was positive in $48.3 \%$ of the patients. Table shows the non-neurological manifestations. Regarding the neurological manifestations, 23 patients $(79.3 \%)$ had parenchymal involvement (hemiparesis $(n=6)$, 
brainstem involvement $(n=1)$, encephalopathy $(n=4)$, optic neuropathy $(n=3)$, dysphasia $(n=1)$, polyneuropathy $(n=6)$, cognitive impairment $(n=4)$, and non-steroidal psychosis $(n=1)$, while the remaining 6 patients $(20.7 \%)$ presented aseptic meningitis as a non-parenchymal affectation (Table). Prior to BT, patients had received the following treatment: oral prednisone $(n=27)$, methylprednisolone bolus $(n=9)$, CsA $(n=8)$, AZA $(n=16)$, MTX $(n=14)$ and mycophenolate $(n=2)$.

After a median of 31 [10-60] months since the beginning of the neurological symptoms, the following BT was initiated: infliximab (IFX) $(n=17)$, adalimumab $(\operatorname{ADA})(n=7)$, tocilizumab (TCZ) $(n=2)$, golimumab (GOL) $(n=2)$ and Etanercept $(E T N)(n=1)$. A first switch to ADA was necessary in 8 patients with IFX due to primary failure. In addition, 2 of them needed a second switch to TCZ, getting a partial response. The BT was discontinued in 5 patients, 2 of them for obtaining clinical remission and the remaining 3 for inefficacy.

After a median follow-up of $5.4 \pm 4.6$ years, complete response was obtained in 15 patients, partial response in 11 and no response in the remaining 3 . We observed an anaphylactic reaction and psoriasis induced by IFX, without other serious adverse events (Table).

Conclusion: BT, especially anti-TNF, seems effective and safe for treatment in NB.

Table.

\begin{tabular}{|c|c|}
\hline \multicolumn{2}{|l|}{ Demographical parameter } \\
\hline - Patients, n & 29 \\
\hline$-\operatorname{Sex}, n(x)$ & $1 S_{\ell} / 14_{9}(51.7 / 48.3)$ \\
\hline - Age, year: (mean 150$)$ & $39.6 \pm 10.5$ \\
\hline - HLA-BS1+ & $14\{48.3\}$ \\
\hline \multicolumn{2}{|l|}{ Non Neurological manifestation } \\
\hline Oral ukers & $28(96.6)$ \\
\hline - Genital ukers & $23(79.3\}$ \\
\hline - Uveitis & $16\{55.2\}$ \\
\hline - Skin lesions & $20(69)$ \\
\hline - Arthritis & $6\{20.7\}$ \\
\hline - Arthralgis & $19(65.6)$ \\
\hline - Venous/arterial thrombosis & $7(24.1) / 3\{10.3\}$ \\
\hline \multicolumn{2}{|l|}{ Neurological manifestation } \\
\hline Parenchymal Disease, $n$ (56) & $23(79.3)$ \\
\hline Henipuresis & $6(20.7)$ \\
\hline - brainstem syndrome & $1(3.4)$ \\
\hline - encephropsthy & $4(13.8)$ \\
\hline - optic neuroputhy & $2(6.8)$ \\
\hline - dysphrsis & $1\{3,4\}$ \\
\hline - polyneuropsthy & $6(20.7)$ \\
\hline - cognitive dysfunction & $4\{13.8\}$ \\
\hline - psychosis & $1(3.4)$ \\
\hline Non-parenchymol Disease (septic & $6 ! 20,7)$ \\
\hline Time from diagnosis to $\mathrm{BT}$ onset, months & $31|10-60|$ \\
\hline (median[1QR]) & \\
\hline \multicolumn{2}{|l|}{ Previous immunosuppressants } \\
\hline - Oral corticoids & $27\{93.1\}$ \\
\hline - Bolus MTP & $9(31)$ \\
\hline - Azathioprine & $16(55.2)$ \\
\hline - Methotrexate & $14\{48.3\}$ \\
\hline - Cyclosporine & $8(27.6)$ \\
\hline - Mycophenolute & $2(6.9)$ \\
\hline \multicolumn{2}{|l|}{ First Biological the rapy } \\
\hline - Inflicimub & $17(58.6)$ \\
\hline - Adalimumub & $7(24.1)$ \\
\hline - Tocilizumub & $2(6.9)$ \\
\hline - Golimumab & 2 (6.9\} \\
\hline - Etanercep: & $1(3.4)$ \\
\hline Follow-up, months (median[1QR]) & $22[18.5 \cdot 74]$ \\
\hline $1^{2} / 2^{N 2}$ Switch, $n(8 / 9)$ & $8\{27.6\} / 2\{6.9\}$ \\
\hline - Oiscontinued (inelficary) & $3(10.3)$ \\
\hline - Severe odverse events" & $0(0)$ \\
\hline
\end{tabular}

Disclosure of Interests: José Luis Martín-Varillas: None declared, Iñigo González-Mazón: None declared, Belén Atienza-Mateo: None declared, Monica Calderón-Goercke: None declared, D. Prieto-Peña: None declared, Lara Sánchez Bilbao: None declared, Vanesa Calvo-Río: None declared, Santos Castañeda Consultant for: Amgen, BMS, Pfizer, Lilly, MSD, Roche, Sanofi, UCB, Esther Vicente: None declared, Olga Maiz: None declared, Clara Moriano: None declared, Elvira Diez Alvarez: None declared, José Luis Andréu Sánchez: None declared, Concepción Delgado Beltrán: None declared, Marta Loredo Martínez: None declared, J. Narváez Consultant for: Bristol-Myers Squibb, Angel Ramos Calvo: None declared, Francisca Sivera: None declared, Enrique Raya: None declared, Norberto Ortego: None declared, Jose Luis Callejas-Rubio: None declared, Anahy Brandy-Garcia: None declared, Alejandro Olive: None declared, Sabela Fernández: None declared, Ricardo Gómez de la Torre: None declared, Ignacio Torre-Salaberri: None declared, Julio Sánchez: None declared, ANA URRUTICOECHEA-ARANA: None declared, Eva SalgadoPérez: None declared, Rafael Melero: None declared, Olga Martínez González: None declared, Susana Romero-Yuste: None declared, Miguel A González-Gay Grant/research support from: Prof. MA Gonzalez-Gay received grants/research supports from Abbvie, MSD, Jansen and Roche., Speakers bureau: Consultation fees/participation in company sponsored speaker's bureau from Pfizer, Lilly, Sobi, Celgene, Novartis, Roche and Sanofi., Ricardo Blanco Grant/research support from: Abbvie, MSD, and Roche, Consultant for: Abbvie, Pfizer, Roche, Bristol-Myers, Janssen, Speakers bureau: Abbvie, Pfizer, Roche, Bristol-Myers, Janssen DOI: 10.1136/annrheumdis-2019-eular.7591

\section{SAT0236 RELATIONSHIP BETWEEN RITUXIMAB CONCENTRATION AND AUTOANTIBODY LEVELS IN ANTI-NEUTROPHIL CYTOPLASMIC ANTIBODY ASSOCIATED VASCULITIS}

Amina Bensalem ${ }^{1}$, David Ternant ${ }^{1,2}$, Nicolas Azzopardi $^{1,3}$, Gilles Paintaud $^{1,2}$ Valérie Gouilleux-Gruart ${ }^{1,4}$, Divi Cornec ${ }^{5,6}$, Ulrich Specks ${ }^{7,}{ }^{1,8}$ Denis Mulleman, The rituximab vs cyclophosphamide for ANCA-associated vasculitis (RAVE) trial. ${ }^{1}$ University of Tours, EA 7501, GICC, Tours, France; ${ }^{2}$ CHRU de Tours, Laboratoire de Pharmacologie-Toxicologie, Tours, France; ${ }^{3} \mathrm{CNRS}$, ERL 7001, Tours, France; ${ }^{4} \mathrm{CHRU}$ de Tours, Laboratoire d'Immunologie, Tours, France; ${ }^{5}$ University of Brest, INSERM U1227, Brest, France; ${ }^{6}$ CHRU de Brest, Service de Rhumatologie, Brest, France; ${ }^{7}$ Mayo Clinic, Division of Pulmonary and Critical Care Medicine, Rochester, United States of America; ${ }^{8} \mathrm{CHRU}$ de Tours, Service de Rhumatologie, Tours, France

Background: Rituximab is approved in patients with anti-neutrophil cytoplasmic antibody (ANCA) associated vasculitis (AAV). Antibodies to protei nase 3 (PR3-ANCA) or myeloperoxidase (MPO-ANCA) decrease with therapy (1) and may therefore be used as biomarkers.

Objectives: To investigate the relationship between rituximab concentration and ANCA levels in AAV patients.

Methods: 92 AAV patients from the RAVE trial (rituximab for ANCA associated vasculitis) were assessed. Both MPO-ANCA, and PR3-ANCA antibody levels were used as biomarkers. ANCA levels were measured at baseline, at months $1,2,4,6,9,18$, and every 6 months until the second rituximab cycle if any, using ELISA supplied by Euroimmun. A semimechanistic model including a deep compartment which was sensitive to rituximab and a feedback mechanism, and a blood compartment were tested to describe the concentration-ANCA autoantibodies relationship. A population modeling approach using sequential methods was used for model building. Influence of sex, body surface area (BSA), BVAS/WG score, newly diagnosed status, major renal involvement at baseline, and relapse were investigated as covariates on pharmacodynamics parameters.

Results: A two compartment model including feedback mechanism from the circulating ANCA well described the concentration autoantibody relationship. The mean (interindividual standard deviation) estimated rituximab IC50, assessing potency, in patients with MPO-ANCA and PR3-ANCA were $21.9 \mathrm{mg} / \mathrm{l}$ (28\%) and $11.2 \mathrm{mg} / \mathrm{l}$ (48.6\%), respectively. Moreover, among patients with PR3-ANCA, rituximab IC50 was higher in patients with major renal involvement at baseline $\left(\mathrm{p}=2.47 \times 10^{-2}\right)$ and newly diagnosed status $\left(p=6.42 \times 10^{-5}\right)$ than in others.

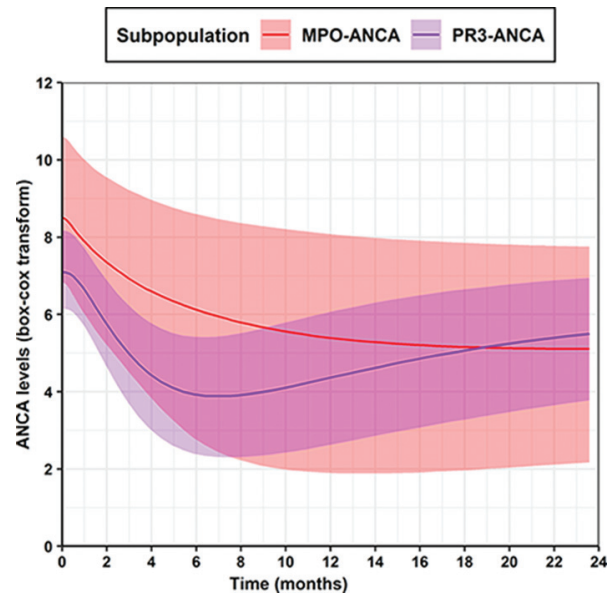

Figure 1. Simulations of ANCA-time profile in patients with MPO-ANCA (red line) and PR3ANCA (purple line), using typical pharmacodynamic parameters. The central curve are the median dynamics of ANCA and the shadow are $90 \%$ prediction intervals. 